Pacific Journal of Mathematic 


\title{
GELFAND AND WALLMAN-TYPE COMPACTIFICATIONS
}

\author{
Charles M. Biles
}

\begin{abstract}
In this paper we compare the Gelfand and Wallman methods of constructing a compactification for a Tychonoff space $X$ from a suitable ring of continuous real-valued functions on $X$. Every Hausdorff compactification $T$ of $X$ is Gelfand constructable; in particular, $T$ is equivalent, as a compactification of $X$, to the structure space of all maximal ideals of the ring of all continuously extendable functions from $X$ to $T$. However, Wallman's method applied to this ring may not yield $T$. We thus inquire into some relationships that exist between the Wallman and Gelfand compactification of $X$ constructed from a suitable ring of functions on $X$.
\end{abstract}

o. Topological preliminaries. All topological spaces in this paper are assumed to be completely regular and Hausdorff. We shall be concerned with methods of constructing compactifications for such spaces.

Let $X$ be a topological space. The space $T$ is an extension of $X$ means there exists a homeomorphism $h$ from $X$ into $T$ such that $h[X]$ is dense in $T$. The function $h$ is called an embedding. Occasionally the necessary embedding maps will be explicitly mentioned, but usually they will be tacitly assumed. In fact, when $T$ is given as an extension of $X$, we may take $X$ as a subspace of $T$. The space $T$ is a compactification of $X$ (denoted $T \in c X$ ) means that $T$ is a compact extension of $X$. The compactifications $T$ and $K$ of a space $X$ are equivalent as compactifications of $X$ (denoted $T=K$ ) means there exists a homeomorphism between $T$ and $K$ such that $h(x)=x$ for each $x \in X$.

We shall use the standard notations [4] regarding $C(X)$, the ring of continuous real-valued functions. For any $f \in C(X)$,

$$
Z(f)=\{x \in X \mid f(x)=0\}
$$

is called the zero-set of $f$. If $\mathscr{C}$ is a subring of $C(X)$, we define $Z[\mathscr{A}]=\{Z(f) \mid f \in \mathscr{A}\}$; however, $Z[C(X)]$ is customarily denoted by $Z(X)$. We shall only refer to subrings of $C(X)$ with unity.

Let $\mathscr{A}$ be a subring of $C(X)$. We shall denote the space of maximal ideals of $\mathscr{A}$ with the Stone topology $[4,7 \mathrm{M}]$, also called the structure space of $\mathscr{A}$, by $H[\mathscr{A}]$. The space of ultrafilters of $Z[\mathscr{A}]$ is denoted by $w Z[\mathscr{A}]$. This space of ultrafilters is constructed by Wallman's method [1] [2]. We shall be primarily concerned with those subrings $\mathscr{A}$ of $C(X)$ for which $w Z[\mathscr{A}] \in c X$ and how these 
subrings relate to a certain type of "structure space" for $\mathscr{A}$.

Let $\mathscr{L}$ be a collection of subsets of $X$. Then $\mathscr{L}$ is a lattice on $X$ means

(1) $\varnothing, X \in \mathscr{L}$;

(2) if $A, B \in \mathscr{L}$, then $A \cap B \in \mathscr{L}$ and $A \cup B \in \mathscr{L}$.

A set in $\mathscr{L}$ is referred to as an $\mathscr{L}$-set.

The lattice $\mathscr{L}$ on $X$ is a Wallman base on $X$ means

(1) $\mathscr{L}$ is a base for the closed subsets of $X$;

(2) $\mathscr{L}$ is a disjunctive lattice on $X$ (i.e., if $A \in \mathscr{L}$ and $x \in$ $X-A$, then there exists $B \in \mathscr{L}$ such that $x \in B$ and $A \cap B=\varnothing)$;

(3) $\mathscr{L}$ is a normal lattice on $X$ (i.e., for each $A, B \in \mathscr{L}$, if $A$ and $B$ are disjoint, then there exists $C, D \in \mathscr{L}$ such that $X-A \subset C$, $X-B \subset D$ and $C \cup D=X)$.

For any lattice $\mathscr{L}$ on $X$, an $\mathscr{L}$-filter is a nonvoid subset $\mathscr{F}$ of $\mathscr{L}$ such that

(1) $\varnothing \notin \mathscr{F}$;

(2) if $A, B \in \mathscr{F}$, then $A \cap B \in \mathscr{F}$;

(3) if $A \in \mathscr{F}, B \in \mathscr{L}$ and $A \subset B$, then $B \in \mathscr{F}$.

An $\mathscr{L}$-ultrafilter is a maximal (with respect to inclusion) $\mathscr{L}$-filter. The set of all $\mathscr{L}$-ultrafilters is denoted by $w \mathscr{L}$.

Let $\mathscr{L}$ be a lattice on $X$. In order to topologize $w \mathscr{L}$, define $A^{*}=\{\mathscr{U} \in w \mathscr{L} \mid A \in \mathscr{Q}\}$ for each $A \in \mathscr{L}$. Then $\left\{A^{*} \mid A \in \mathscr{L}\right\}$ is a base for the closed sets of some (necessarily unique) topology for $w \mathscr{L}$. We shall only consider $w \mathscr{L}$ with this topology. Now $w \mathscr{L} \in c X$ if and only if $\mathscr{L}$ is a Wallman base on $X$ (with respect to the embedding $\operatorname{map} \varphi: X \rightarrow w \mathscr{L}$ defined by $\varphi(x)=\{A \in \mathscr{L} \mid x \in A\})$. If $T \in c X$, then $T$ is a Wallman-type compactification of $X$ means there exists a Wallman base $\mathscr{L}$ on $X$ such that $T=w \mathscr{L}$. It is unknown whether or not every compactification is Wallman-type. If $T \in c X$, then $T$ is a $z$-compactification of $X$ means there exists a Wallman base $\mathscr{L} \subset Z(X)$ such that $T=w \mathscr{L}$.

1. Filter ideals. Let $X$ be a topological space and $\mathscr{A}$ a subring of $C(X)$.

Definition 1.1. The ideal $I$ of $\mathscr{A}$ is a filter ideal of $\mathscr{A}$ means $Z[I]$ is a $Z[\mathscr{A}]$-filter. The set of all maximal filter ideals is denoted by $F[\mathscr{A}]$.

DeFinition 1.2. $\mathscr{A}$ is a wallman subring of $C(X)$ means that $Z[\mathscr{A}]$ is a Wallman base on $X$.

We first give some elementary facts about filter ideals, the proofs of which are straight forward. 
Proposition 1.3. The ideal $I$ is a filter ideal of $\mathscr{A}$ if and only if $Z(f) \neq \varnothing$ for each $f \in I$.

Thus an ideal of $\mathscr{A}$ need not be a filter ideal. Further, every ideal of $\mathscr{A}$ is a filter ideal if and only if $\mathscr{A}$ is inverse closed (if $f \in \mathscr{A}$ and $Z(f)=\varnothing$, then $1 / f \in \mathscr{A})$.

Proposition 1.4. If $F$ is a $Z[\mathscr{A}]$-filter, then

$$
Z^{\leftarrow}[F]=\{f \in \mathscr{A} \mid Z(f) \in F\}
$$

is a filter ideal of $\mathscr{A}$.

A filter ideal $I$ of $\mathscr{A}$ is a $z$-filter ideal means if $f \in \mathscr{A}$ and $Z(f) \in Z[I]$, then $f \in I$. Then there is a one-to-one correspondence between the $Z[\mathscr{A}]$-filters and the $z$-filter ideals of $\mathscr{A}$. The next two propositions show that there is also a one-to-one correspondence between $Z[\mathscr{A}]$-ultrafilters and maximal filter ideals.

Proposition 1.5. If $I$ is a maximal filter ideal in $\mathscr{A}$, then $Z[I] \in w Z[\mathscr{A}]$.

Proof. Now $Z[I]$ is a $Z[\mathscr{A}]$-filter. Suppose $F$ is a $Z[\mathscr{A}]-$ filter such that $Z[I] \subset F$. Then $Z[F]$ is a filter ideal of $\mathscr{A}$ and $I \subset Z^{\leftarrow}[Z[I]] \subset Z^{\leftarrow}[F]$. Since $I$ is a maximal filter ideal, then $I=Z^{\leftarrow}[F]$. Thus $Z[I]=F$; hence, $Z[I] \in w Z[\mathscr{A}]$.

Proposition 1.6. If $\mathscr{U} \in w Z[\mathscr{A}]$, then $Z[\mathscr{U}]$ is a maximal filter ideal.

Proof. Since $\mathscr{U} \in w Z[\mathscr{A}]$, then $Z[\mathscr{U}]$ is a filter ideal by 1.4 . Suppose $I$ is an ideal of $\mathscr{A}$ such that $Z[\mathscr{U}] \subset I$. Then $\mathscr{U} \subset Z[I]$ where $Z[I]$ is a $Z[\mathscr{A}]$-filter by 1.3 . Since $\mathscr{C}$ is maximal, then $\mathscr{C}=Z[I]$. So $I \subset Z^{-}[Z[I]]=Z \leftarrow[\mathscr{U}]$; thus $I=Z^{-}[\mathscr{U}]$. Hence, $Z-[\mathscr{U}]$ is a maximal filter ideal.

Proposition 1.7. Every maximal filter ideal of $\mathscr{A}$ is a prime ideal of $\mathscr{A}$.

Proof. Let $I$ be a maximal filter ideal of $\mathscr{A}$ and suppose $I$ is not prime. We select $f, g \in \in \mathscr{A}$ such that $f g \in I$, but $f \notin I$ and $g \notin I$. So $I$ is properly contained in the ideals $I_{1}=I+\mathscr{A} f$ and $I_{2}=I+\mathscr{A} g$. Since $I_{1}, I_{2}$ are not filter ideals, by 1.1 we select $h_{1}, h_{2} \in I$ and $k_{1}, k_{2} \in$ \&f such that $Z\left(h_{1}-k_{1} f\right)=\varnothing$ and $Z\left(h_{2}-k_{2} g\right)=\varnothing$. Clearly $h_{1}-$ $k_{1} f \in I_{1}$ and $h_{2}-k_{2} g \in I_{2}$. Since $\left(Z\left(h_{1}\right) \cap Z\left(k_{1}\right)\right) \cup\left(Z\left(h_{1}\right) \cap Z(f)\right)=\varnothing$ and 
$\left(Z\left(h_{2}\right) \cap Z\left(k_{2}\right)\right) \cup\left(Z\left(h_{2}\right) \cap Z(g)\right)=\varnothing$, then $Z\left(h_{1}\right) \cap Z\left(h_{2}\right) \cap Z(f g)=\varnothing$ so, $Z\left(h_{1}^{2}+h_{2}^{2}+(f g)^{2}\right)=\varnothing$. But $h_{1}^{2}+h_{2}^{2}+(f g)^{2} \in I$, contradicting $I$ is a filter ideal by 1.1. Hence, $I$ must be a prime ideal of $\mathscr{A}$.

The following easily proved characterization of maximal filter ideals we state without proof:

Proposition 1.8. Let $M$ be a filter ideal of $\mathscr{A}$. Then $M \in F[\mathscr{A}]$ if and only if for every $f \in \mathscr{A}-M$ there exists $g \in M$ such that $Z(f) \cap Z(g)=\varnothing$.

2. Maximal filter ideal spaces. Let $X$ be a topological space. Let $\mathscr{A}$ be a subring of $C(X)$ (we shall only refer to subrings of $\mathscr{A}$ with unity). We denote the structure space of $\mathscr{A}$ by $H[\mathscr{A}]$ (see $[4,7 \mathrm{M}]$ ) and the set of maximal filter ideals of $\mathscr{A}$ by $F[\mathscr{A}]$. We seek to define a "structure space" topology for $F[\mathscr{A}]$ and to examine the relationships between the spaces $F[\mathscr{A}]$ and $w Z[\mathscr{Z}]$. In particular, we show $F[\mathscr{A}]=w Z[\mathscr{A}]$ equivalent as compactifications of $X$ ) if and only if $Z[\mathscr{A}]$ is a Wallman base on $X$. Furthermore, $F[\mathscr{A}]$ is a compactification of $X$ if and only if $Z[\mathscr{A}]$ is a Wallman base on $X$. Accordingly, we shall refer to $\mathscr{A}$ as a Wallman ring on $X$ if $Z[\mathscr{A}]$ is a Wallman base on $X$.

THEOREM 2.1. Let $X$ be a topological space and $\mathscr{A}$ a subring of $C(X)$. For each $x \in X$ define $M_{x}=\{f \in \mathscr{A} \mid f(x)=\varnothing\}$. Then

(a) $M_{x} \in F[\mathscr{A}]$ for each $x \in X$ if and only if $Z[\mathscr{A}]$ is a disjunctive lattice on $X$;

(b) If $Z[\mathscr{A}]$ is a disjunctive lattice on $X$, then the mapping $x \rightarrow M_{x}$ is one-to-one if and only if $\mathscr{A}$ strongly separates points in $X$ (i.e., if $x, y \in X, x \neq y$, then there exists $f \in \mathscr{A}$ such that $f(x)=0$ and $f(y) \neq 0$ ).

Proof. (a) Suppose $M_{x} \in F[\mathscr{A}]$ for each $x \in X$. Let $A \in Z[\mathscr{A}]$ and $x \in X-A$. Select $f \in \mathscr{A}$ such that $A=Z(f)$. Since $f \in \mathscr{A}-M_{x}$, then by 1.8 we may choose $g \in M_{x}$ such that $Z(f) \cap Z(g)=\varnothing$. Then $Z(g) \in Z[\mathscr{A}], x \in Z(g)$ and $Z(g) \cap A=\varnothing$. Hence, $Z[\mathscr{A}]$ is a disjunctive lattice on $X$. Conversely, suppose $Z(\mathscr{A})$ is disjunctive. By 1.3 , $M_{x}$ is a filter ideal of $\mathscr{A}$ for each $x \in X$. Suppose $x \in X$. Let $I$ be a filter ideal of $\mathscr{A}$ properly containing $M_{x}$ and select $f \in I-M_{x}$. Since $Z[\mathscr{A}]$ is disjunctive, select $Z(g) \in Z[\mathscr{A}]$ such that $x \in Z(g)$ and $Z(g) \cap Z(f)=\varnothing$. Then $g \in M_{x}$, so $g \in I$, and thus $f^{2}+g^{2} \in I$, contradicting 1.3. Hence, $M_{x} \in F[\mathscr{A}]$.

(b) Since $Z[\mathscr{A}]$ is a disjunctive lattice on $X$, then $M_{x} \in F[\mathscr{A}]$ for each $x \in X$. Suppose the mapping $x \rightarrow M_{x}$ is one-to-one. Let 
$x, y \in X$ such that $x \neq y$. Then $M_{x} \neq M_{y}$. So there exists $f \in \mathscr{A}$ such that $f(x) \neq 0$ and $f(y)=0$. So $\mathscr{A}$ strongly separates points in $X$. The converse is obvious. This completes the proof.

We now put a structure space topology on $F[\mathscr{A}]$. For each $f \in \mathscr{A}$, define $f^{*}=\{I \in F[\mathscr{A}] \mid f \in I\}$. Easily $0^{*}=F[\mathscr{A}]$ and $f^{*}=$ $\varnothing$ whenever $Z(f)=\varnothing$. Since every maximal filter ideal is prime, then $(f g)^{*}=f^{*} \cup g^{*}$. Hence, $\left\{f^{*} \mid f \in \mathscr{A}\right\}$ defines a base for some topology (necessarily unique) on $F[\mathscr{A}]$. We shall only consider this topology on $F[\mathscr{A}]$. Easily $\{I\}=\cap\left\{f^{*} \mid f \in I\right\}$ for each $I \in F[\mathscr{A}]$; hence, $F[\mathscr{A}]$ is a $T_{1}$-space.

\section{THEOREM 2.2. $F[\mathscr{A}]$ is compact.}

Proof. Let $\mathscr{K}$ be a nonvoid collection of nonvoid basic closed subsets of $F[\mathscr{A}]$ with the finite intersection property. Let $\mathscr{K}^{\prime}=$ $\left\{Z(f) \mid f \in \mathscr{A}, f^{*} \in \mathscr{K}\right\}$. Then $\mathscr{K}^{\prime}$ is a nonempty collection of zero sets of $\mathscr{A}$ with the finite intersection property. So we may select $\mathscr{U} \in w Z[\mathscr{A}]$ such that $\mathscr{K}^{\prime} \subset \mathscr{U}$. For each $f \in \mathscr{A}$ where $f^{*} \in \mathscr{K}$, we have $Z(f) \in \mathscr{K}^{\prime} \subset \mathscr{U} \Rightarrow f \in Z^{\leftarrow}[\mathscr{U}] \in F[\mathscr{A}]$ (by 1.6) $\rightarrow Z^{\leftarrow}[\mathscr{U}] \in f^{*}$; thus, $Z[\mathscr{U}] \in \cap \mathscr{K}$. Hence, $F[\mathscr{A}]$ is compact.

We now seek conditions under which $F[\mathscr{A}]$ is a compactification of $X$ with respect to the mapping $x \rightarrow M_{x}(=\{f \in \mathscr{A} \mid f(x)=0\})$. By 2.1, we must have a subring $\mathscr{A}$ of $C(X)$ such that $\mathscr{A}$ strongly separates points of $X$ and $Z[\mathscr{A}]$ is a disjunctive lattice on $X$.

THEOREM 2.3. $F[\mathscr{A}]$ is Hausdorff if and only if $F_{1}, F_{2} \in F[\mathscr{A}]$, $F_{1} \neq F_{2} \rightarrow$ there exists $f, g \in \mathscr{A}$ such that $(f g)^{*}=F[\mathscr{A}], f \notin F_{1}$ and $g \notin F_{2}$.

Proof. Suppose $F[\mathscr{A}]$ is Hausdorff. Let $F_{1}, F_{2} \in F[\mathscr{A}], F_{1} \neq$ $F_{2}$. Select $f, g \in \mathscr{A}$ such that $F_{1} \in F[\mathscr{A}]-f^{*}, F_{2} \in F[\mathscr{A}]-g^{*}$ and $\left(F[\mathscr{A}]-f^{*}\right) \cap\left(F[\mathscr{A}]-g^{*}\right)=\varnothing$. Then $f \notin F_{1}, g \notin F_{2}$ and $f^{*} \cup g^{*}=$ $(f g)^{*}=F[\mathscr{A}]$. Suppose the converse hypothesis holds. Let $F_{1}, F_{2} \in$ $F[\mathscr{A}], F_{1} \neq F_{2}$. Select $f, g \in \mathscr{A}$ such that $f \notin F_{1}, g \notin F_{2}$ and $(f g)^{*}=$ $F[\mathscr{A}]$. Then $F_{1} \in F[\mathscr{A}]-f^{*}, F_{2} \in F[\mathscr{A}]-g^{*}$ and $\left(F[\mathscr{A}]-f^{*}\right) \cap$ $\left(F[\mathscr{A}]-g^{*}\right)=\varnothing$. This completes the proof.

Corollary 2.4. Suppose $Z[\mathscr{A}]$ is a base for the closed subsets of $X$. Then $F[\mathscr{A}]$ is Hausdorff if and only if $F_{1}, F_{2} \in F[\mathscr{A}], F_{1} \neq$ $F_{2} \rightarrow$ there exists $f, g \in \mathscr{A}$ such that $f \notin F_{1}, g \notin F_{2}$ and $f g=0$.

THEOREM 2.5. Let $\mathscr{A}$ be a subring of $C(X)$ such that $Z[\mathscr{A}]$ is 
a disjunctive lattice on $X$. Let $\varphi$ denote the mapping $x \rightarrow M_{x}$ from $X$ into $F[\mathscr{A}]$. Then

(a) $\varphi: X \rightarrow F[\mathscr{A}]$ is continuous,

(b) $\varphi[X]$ is dense in $F[\mathscr{A}]$, and

(c) $\varphi$ is a homeomorphism between $X$ and $\varphi[X]$ if and only if $\mathscr{A}$ strongly separates points from the closed sets in $X$ (i.e., if $F$ is a closed subset of $X$ and $x \in X-F$, then there exists $f \in \mathscr{A}$ such that $F \subset Z(f)$ and $f(x) \neq 0)$.

Proof. By 2.1 (a), $M_{x} \in F[\mathscr{A}]$ for every $x \in X$.

(a) Since $\varphi^{-}\left[f^{*}\right]=Z(f)$ for each $f \in \mathscr{A}$, it becomes straightforward to show $\varphi: X \rightarrow F[\mathscr{A}]$ is continuous.

(b) Let $f \in \mathscr{A}$. Then $F[\mathscr{A}]-f^{*}$ is a basic open set in $F[\mathscr{A}]$. Suppose $\left(F[\mathscr{A}]-f^{*}\right) \cap \varphi[X]=\varnothing$. Let $x \in X$. Then $\varphi(x)=M_{x} \notin$ $F[\mathscr{A}]-f^{*}$, so $M_{x} \in f^{*}$. Thus $f \in M_{x}$ for every $x \in X$; i.e., $f=0$. So $f^{*}=F[\mathscr{A}]$. Hence, every nonvoid basic open set of $F[\mathscr{A}]$ intersects $\varphi[X]$; i.e., $\varphi[X]$ is dense in $F[\mathscr{A}]$.

(c) First, suppose $\mathscr{A}$ strongly separates points and closed sets in $X$. Then $Z[\mathscr{A}]$ is a base for the closed sets in $X$. Since

$$
\varphi^{-}\left[f^{*} \cap \varphi[X]\right]=Z(f)
$$

for each $f \in \mathscr{A}$, then $\varphi$ and $\varphi^{\leftarrow}$ are continuous. By $2.1(\mathrm{~b}), \varphi$ is one-to-one. Hence, $\varphi$ is a homeomorphism between $X$ and $\varphi[X]$. Let $F$ be a closed subset of $X$. Then $\varphi[F]$ is a closed subset of $\varphi[X]$. So we may select $\mathscr{K} \subset \mathscr{A}$ such that

$$
\varphi[F]=\cap\left\{f^{*} \cap \varphi[X] \mid f \in \mathscr{K}\right\} .
$$

Thus $F=\cap\left\{\varphi^{-}\left[f^{*} \cap \varphi[X]\right] \mid f \in \mathscr{K}\right\}=\cap\{Z(f) \mid f \in \mathscr{K}\}$; so $Z[\mathscr{A}]$ is a base for the closed subsets of $X$. Hence, $\mathscr{A}$ strongly separates points from closed sets in $X$.

Let $\mathscr{A}$ be a subring of $C(X)$ which strongly separates points from closed sets in $X$ and for which $Z[\mathscr{A}]$ is disjunctive. Then the mapping $\varphi: X \rightarrow F[\mathscr{A}]$ defined by $\varphi(x)=M_{x}$ embeds $X$ into the compact $T_{1}$-space $F[\mathscr{A}]$. Define $h: X \rightarrow w Z[\mathscr{A}]$ by $h(x)=\mathscr{U}_{x}(=$ $\{A \in Z[\mathscr{A}] \mid x \in A\})$. By [2, Th. 2.7], $h$ embeds $X$ into the compact $T_{1}$-space $w Z[\mathscr{A}] . \quad$ Define $H: w Z[\mathscr{A}] \rightarrow F[\mathscr{A}]$ by $H(\mathscr{U})=Z[\mathscr{U}]$ for each $\mathscr{Q} \in w Z[\mathscr{A}]$.

THEOREM 2.6. The mapping $H$ is a homeomorphism between $w Z[\mathscr{A}]$ and $F[\mathscr{A}]$.

Proof. By 1.5 and 1.6, $H$ is a bijection. Now $\left\{Z(f)^{*} \mid f \in \mathscr{A}\right\}$, where $Z(f)^{*}=\{\mathscr{C} \in w Z[\mathscr{A}] \mid Z(f) \in \mathscr{Q}\}$, is a base for the closed sets 
of $w Z[\mathscr{A}]$ (see [1] or [2]). Since $H\left[Z(f)^{*}\right]=f^{*}$ for each $f \in \mathscr{A}$, then both $H$ and $H^{-}$are continuous. Hence, $H$ is a homeomorphism.

Theorem 2.7. $F[\mathscr{A}] \in c X$ if and only if $\mathscr{A}$ is a Wallman ring.

Proof. By 2.6, $H$ defines a homeomorphism between $F[\mathscr{A}]$ and $w Z[\mathscr{A}]$. But $w Z[\mathscr{A}] \in c X$ if and only if $Z[\mathscr{A}]$ is a Wallman base on $X$. Hence, $F[\mathscr{A}] \in c X$ if and only if $\mathscr{A}$ is a Wallman ring.

Hence, the structure space $F[\mathscr{A}]$ of the maximal filter ideals of a subring $\mathscr{A}$ of $C(X)$ is a (Hausdorff) compactification if and only if $\mathscr{A}$ is a Wallman ring. Moreover, $F[\mathscr{A}]$ is a Wallman-type compactification of $X$.

3. Maximal ideal spaces and maximal filter ideal spaces. In this section $\mathscr{A}$ is a subring of $C(X)$ containing $\mathscr{R}$, the constant real-valued functions on $X$. For $x \in X$, define $M_{x}=\{f \in \mathscr{A} \mid f(x)=0\}$. The mapping $f+M_{x} \rightarrow f(x)$ is a ring isomorphism between $\mathscr{A} / M_{x}$ and $\mathscr{R}$; so, $M_{x} \in H[\mathscr{A}]$ for each $x \in X$. Similarly, $M_{x} \in F[\mathscr{A}]$ for each $x \in X$ (1.3). We topologize $H[\mathscr{A}]$ by taking the set of all $f^{\sharp}=\{M \in H[\mathscr{A}] \mid f \in M\}, f \in \mathscr{A}$, as a base for the closed sets; i.e., $H[\mathscr{A}]$ is the structure space of $\mathscr{A}[4,7 \mathrm{M}]$. Similarly we topologize $F[\mathscr{A}]$, where a basic closed set is denoted $f^{*}=\{F \in F[\mathscr{A}] \mid f \in F\}$, $f \in \mathscr{A}$. Define the mapping $\varphi: X \rightarrow F[\mathscr{A}]$ by $\varphi(x)=M_{x}$ and $\psi: X \rightarrow$ $H[\mathscr{A}]$ by $\psi(x)=M_{x}$. We obtain $\varphi[Z(f)]=f^{\sharp} \cap \varphi[X]$ and $\psi[Z(f)]=$ $f^{*} \cap \psi[X]$. Hence, $H[\mathscr{A}]$ is an extension of $X$ (via $\psi$ ), $F[\mathscr{A}]$ is an extension of $X$ (via $\varphi$ ) if and only if $Z[\mathscr{A}]$ is a base for the closed sets in $X$. Now $F[\mathscr{A}]$ and $H[\mathscr{A}]$ are both compact $T_{1}$-spaces [see 2.2 and $4,7 \mathrm{M}]$. From $\S 2, F[\mathscr{A}] \in c X$ if and only if $\mathscr{A}$ is a Wallman ring on $X$. From $[4,7 \mathrm{M}], H[\mathscr{A}] \in c X$ if and only if $Z[\mathscr{A}]$ is a base for the closed subsets of $X$ and $H[\mathscr{A}]$ is Hausdorff.

We remark that even if both $H[\mathscr{A}]$ and $F[\mathscr{A}] \in c X$, they need not yield equivalent compactifications of $X$. For example, let $X=\mathscr{R}$ (reals with the usual topology) and $\mathscr{R}^{*}$ be the one-point compactification of $\mathscr{R}$. Let $\mathscr{A}$ be the ring of all functions in $C(\mathscr{R})$ having continuous extensions to $\mathscr{R}^{*}$. Then $\mathscr{A}$ is a Wallman ring and $F[\mathscr{A}]=w Z[\mathscr{A}]=\beta \mathscr{R}$, but $H[\mathscr{A}]=\mathscr{R}^{*}$. This situation generalizes to arbitrary locally compact Lindelof spaces [1] [5]. However, $F\left[C^{*}(X)\right]=w Z(X)=\beta X=H\left[C^{*}(X)\right]$. Thus, we inquire into possible relationships between $F[\mathscr{A}]$ and $H[\mathscr{A}]$.

We first present the following analogue of the Gelfand-Komolgoroff Theorem [4, 7.3] which yields a representation theorem for the maximal filter ideals of $\mathscr{A}$ when $w Z[\mathscr{A}] \in c X$. 
THEOREm 3.1. Let $\mathscr{A}$ be a Wallman ring on the space $X$ and $T=w Z[\mathscr{A}]$. The maximal filter ideals in $\mathscr{A}$ are then given by $F^{t}=\left\{f \in \mathscr{A} \mid t \in c 1_{T} Z(f)\right\} \quad(t \in T)$.

Proof. Let $t \in T$. Easily $F^{t}$ is an ideal. From 1.3, $F^{t}$ is a filter ideal. We now show $F^{t} \in F[\mathscr{A}]$. Suppose $F \in F[\mathscr{A}]$ such that $F^{t} \subset F$ and $F^{t} \neq F$. Select $f \in F$ such that $t \notin c 1_{T} Z(f)$. Since $T=$ $w Z[\mathscr{A}]$, select $g \in \mathscr{A}$ such that $t \in c 1_{T} Z(g)$ and $Z(f) \cap Z(g)=\varnothing$. But then $f, g \in F$ and $Z(f) \cap Z(g)=\varnothing$, contradicting $F \in F[\mathscr{A}]$. So $F^{t}$ is maximal. It remains to show that if $F \in F[\mathscr{A}]$, then $F=F^{t}$ for some $t \in T$. Let $F \in F[\mathscr{A}]$. Then $Z[F] \in w Z[\mathscr{A}]$, so

$$
\cap\left\{\mathrm{c} 1_{T} Z(f) \mid f \in F\right\}=\{t\}
$$

for some $t \in T[1],[6]$. Hence, $F=F^{t}$. This completes the proof.

The above theorem also yields an explicit one-to-one correspondence between the points of $T$ and the maximal filter ideals in $\mathscr{A}$.

Since $C(X)$ is inverse closed and $w Z(X)=\beta X$, we have the

CoRollary 3.2. (Gelfand-Komolgoroff theorem). For any space $X, H[C(X)]=F[C(X)]=w Z(X)=\beta X$ and the maximal ideals of $C(X)$ are given by $M^{t}=\left\{f \in C(X) \mid t \in \mathrm{c1}_{\beta_{X}} Z(f)\right\}$.

Now, since $Z(X)=Z\left[C^{*}(X)\right]$, then $C^{*}(X)$ is also a Wallman ring on $X$ and $F\left[C^{*}(X)\right]=w Z(X)=\beta X$. Since $H[C(X)]=H\left[C^{*}(X)\right]$ [4, 7.11], then $H\left[C^{*}(X)\right]=F\left[C^{*}(X)\right]$ (i.e., equivalent as compactifications of $X)$.

We now inquire into relationships between maximal ideals and maximal filter ideals.

Theorem 3.3. Suppose $H[\mathscr{A}] \in c X$. Then every maximal filter ideal is contained in a unique maximal ideal.

Proof. Let $F \in F[\mathscr{A}]$. Suppose $M, N \in H[\mathscr{A}]$ where $F \subset M, N$ and $M \neq N$. Select $f, g \in \mathscr{A}$ such that $f g=0, f \notin M$ and $g \notin N$ [4, $7 M]$. But then $f g=0 \in F$ so $f \in F$ or $g \in F(1.7)$; hence, $f \in M$ or $g \in N$. From this contradiction, we conclude $M=N$.

Corollary 3.4. Suppose $H[\mathscr{A}] \in c X$. If each maximal ideal, which contains a maximal filter ideal, contains a unique maximal filter ideal, then $F[\mathscr{A}] \in c X$. 
Proof. Since $H[\mathscr{A}] \in c X$, then $Z[\mathscr{A}]$ is a base for the closed subsets of $X$. It then suffices to show that $F[\mathscr{A}]$ is Hausdorff. Let $F, G \in F[\mathscr{A}], F \neq G$. There exist unique $M, N \in H[\mathscr{A}]$ such that $F \subset M, G \subset N$ (3.3). Since $M \neq N$ by hypothesis, we select $f, g \in \mathscr{A}$ such that $f g=0, f \notin M$ and $g \notin N$. So $f, g \in \mathscr{A}, f g=0, f \notin F$

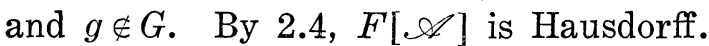

Suppose now that $T \in c X$ and $\mathscr{A}$ is a subring of $E(X, T)$ (the ring of all functions on $X$ continuously extendable to $T$ ) such that $\mathscr{A}$ contains $\mathscr{R}$ (the constant real-valued functions on $X$ ) and $Z[\mathscr{A}$ ] is a base for the closed subsets of $X$. Then $\psi: X \rightarrow H[\mathscr{A}]$ and $\varphi: X \rightarrow F[\mathscr{A}]$ embed $X$ as a dense subspace of the compact $T_{1}$-spaces $H[\mathscr{A}]$ and $F[\mathscr{A}]$, respectively.

For $f \in E(X, T)$, denote the continuous extension by $f^{T}$. For $t \in T$, define $M^{t}=\left\{f \in \mathscr{A} \mid f^{T}(t)=0\right\}$. Then $M^{t} \in H[\mathscr{A}]$ for each $t \in T$ since the mapping $f+M^{t} \rightarrow f^{T}(t)$ is a ring isomorphism between $\mathscr{A} / M^{t}$ and $\mathscr{R}$. Thus the mapping $\psi: X \rightarrow H[\mathscr{A}]$ defined by $\psi(x)=$ $M_{x}$ is extendable from $X$ to $T$ by $\psi(t)=M^{t}$. Note that $M^{x}=M_{x}$ for each $x \in X$.

\section{LEMMA 3.5. $\psi^{\leftarrow}\left[f^{\sharp}\right]=Z\left(f^{T}\right)$.}

Proof. $t \in Z\left(f^{T}\right)$ if and only if $f^{T}(t)=0$ if and only if $f \in M^{t}$ if and only if $M^{t} \in f^{\sharp}$ if and only if $\psi(t) \in f^{\#}$ if and only if $t \in \psi^{t}\left[f^{\sharp}\right]$.

Hence, $\psi: T \rightarrow H[\mathscr{A}]$ is continuous. So $\psi[T]$ is a compact subspace of $H[\mathscr{A}]$. We then obtain the

THEOREM 3.6. If $H[\mathscr{A}]$ is Hausdorff, then

(1) $H[\mathscr{A}] \in c X($ via $\psi: T \rightarrow H[\mathscr{A}])$;

(2) $H[\mathscr{A}]=\psi[T]=\left\{M^{t} \mid t \in T\right\}$;

(3) $H[\mathscr{A}] \leqq T$; and

(4) $H[\mathscr{A}]=T$ if and only if $\psi$ is injective if and only if $\left\{f^{T} \mid f \in \mathscr{A}\right\}$ separates points in $T$ if and only if $\left\{Z\left(f^{T}\right) \mid f \in \mathscr{A}\right\}$ is a base for the closed subsets of $T$.

Proof. (1) and (2). Now $\psi[T]=\mathrm{c1}_{H[\aleph]} \psi[T]$ since a compact subspace of a Hausdorff space is closed. Also, $\mathrm{c} 1_{H[\mathscr{N}} \psi[T]=H[\mathscr{A}]$ since $\psi[X]$ is dense in $H[\mathscr{A}]$.

(3). Obvious.

(4). A continuous bijection from a compact space to a Hausdorff space is a homeomorphism.

Theorem 3.7. Suppose $T=F[\mathscr{A}]$. Then $T=H[\mathscr{A}]$ if and only if each maximal ideal contains a unique maximal filter ideal and $H[\mathscr{A}]$ is Hausdorff. 
Proof. Suppose $H[\mathscr{A}]=T$. Let $M^{t} \in H[\mathscr{A}]$. Then $F^{t} \subset M^{t}$, so every maximal ideal contains a maximal filter ideal (3.6 (2)). Since $T=H[\mathscr{A}]$, then $\psi: T \rightarrow H[\mathscr{A}]$ is injective (3.6 (4)). Hence, if $F^{t}, F^{s} \subset M^{p}$ where $t, s, p \in T$ (3.1), then $t=s=p$. So each maximal ideal contains a unique maximal filter ideal. The Hausdorff condition is obvious.

Now assume the converse hypothesis and suppose $H[\mathscr{A}]<T$ $(3.6(3))$. Then $\psi$ is not injective $(3.6(4))$. Select $t, s \in T$ such that $t \neq s$, but $M^{t}=M^{s}$. Since $T=w Z[A]=F[A]$, then $F^{t} \neq F^{s}$ (3.1). Clearly $F^{t} \subset M^{t}$ and $F^{s} \subset M^{s}$. So $F^{t}, F^{s} \subset M^{t}$ and $F^{t} \neq F^{s}$, contradicting our assumption that each maximal ideal contains a unique maximal filter ideal. This completes the proof.

TheOREm 3.8. Suppose $T=H[\mathscr{A}]$. Then $T=F[\mathscr{A}]$ if and only if $\operatorname{cl}_{T} Z(f) \cap \operatorname{cl}_{T} Z(g)=\varnothing$ whenever $Z(f) \cap Z(g)=\varnothing$ and $f, g \in \mathscr{A}$.

Proof. Since $\left\{f^{T} \mid f \in \mathscr{A}\right\}$ is a base for the closed subsets of $T$ (3.6 (4)), then so is $\left\{\mathrm{cl}_{T} Z(f) \mid f \in \mathscr{A}\right\}$. By [1,3.3], $T=w Z[\mathscr{A}]$ if and only if $\operatorname{cl}_{T} Z(f) \cap \operatorname{cl}_{T} Z(g)=\varnothing$ whenever $Z(f) \cap Z(g)=\varnothing$ and $f, g \in \mathscr{A}$. This completes the proof since $F[\mathscr{A}]=w Z[\mathscr{A}](2.6)$.

Hence, if $T \in c X$ is "constructable" as a maximal ideal space of $\mathscr{A}$, where $\mathscr{A}$ is a subring of $E(X, T)$ containing $\mathscr{R}$, then $T$ is also constructable as the ultrafilter space from the zero-sets of $\mathscr{A}$ if and only if disjoint zero-sets of $\mathscr{A}$ have disjoint closures in $T$. Conversely, if $T$ is "constructable" as the ultrafilter space from the zero-sets of $\mathscr{A}$, then $T$ is constructable as the maximal ideal space of $\mathscr{A}$ if and only if each maximal ideal contains a unique maximal filter ideal and the maximal ideal space is Hausdorff.

THEOREM 3.9. Suppose $H[\mathscr{A}]=T$ and $F[\mathscr{A}] \in c X$. Then $T \leqq$ $F[\mathscr{A}]$.

Proof. Let $F \in F[\mathscr{A}]$. Since $T$ is compact and

$$
\mathscr{F}=\left\{\mathrm{cl}_{T} Z(f) \mid f \in F\right\}
$$

is a nonvoid set of nonvoid closed subsets of $T$ with the $f i p$, then $\cap \mathscr{F} \neq \varnothing$. Since $\left\{\mathrm{cl}_{T} Z(f) \mid f \in \mathscr{A}\right\}$ is a base for the closed subsets of $T$, then $\cap \mathscr{F}$ is a singleton (denote $F \rightarrow t$ ). Thus, for each $F \in$ $F[\mathscr{A}]$ there exists a unique $t \in T$ such that $F \rightarrow t$. Define $h: F[\mathscr{A}] \rightarrow$ $T$ by $h(F)=t$ where $F \rightarrow t$. Then $h$ is a surjection and $h\left(F_{x}\right)=x$ for each $x \in X$. Since $h^{\leftarrow}\left[\operatorname{cl}_{T} Z(f)\right]=\cap\left\{g^{*} \mid \operatorname{cl}_{T} Z(f) \subset \operatorname{int}_{T} Z\left(g^{T}\right), g \in \mathscr{A}\right\}$ for each $f \in \mathscr{A}$, then $h$ is continuous. Hence, $T \leqq F[\mathscr{A}]$ (via $h$ ).

Corollary 3.10. Suppose $H[\mathscr{A}]=T$. Then $T=F[\mathscr{A}]$ if and 
only if each maximal ideal contains a unique maximal filter ideal.

Proof. Suppose each maximal filter ideal contains a unique maximal filter ideal. Then $F[\mathscr{A}] \in c X$ by 3.4. The mapping $h: F[\mathscr{A}] \rightarrow$ $T$ defined in the proof of 3.9 is then injective. Hence, $T=F[\mathscr{A}]$. The converse follows from 3.7. This completes the proof.

4. An application to $E(X, T)$. Let $T \in c X$. Easily $Z[E(X, T)]$ is a base for the closed subsets of $X$. In 1964 Frink [3] mentioned that $Z[E(X, T)]$ was a Wallman base on $X$. However, Brooks, in a paper published in 1967 [2], mentioned he could not prove this. Subsequently Hager, in a 1969 paper. provided a "constructive" proof. We offer here a proof that $Z[E(X, T)]$ is a Wallman base on $X$ based on 2.4 and 2.7. We first observe

Lemma 4.1. Suppose $\mathscr{A}$ is a subring of $C(X)$ such that if $f \epsilon$ $\mathscr{A}$, then $|f| \in \mathscr{A}$. Let $I$ be a $z$-filter ideal of $\mathscr{A}$. Then the following are equivalent:

(1) I is a prime ideal of $\mathscr{A}$;

(2) I contains a prime ideal of $\mathscr{A}$;

(3) if $f, g \in \mathscr{A}$ and $f g=0$, then $f \in I$ or $g \in I$; and

(4) for each $f \in \mathscr{A}$ there exists $g \in I$ such that $f$ does not change sign on $Z(g)$.

Proof. The techniques of $[4,2.9]$ apply verbatim.

THEOREM 4.2. Let $\mathscr{A}$ be subring of $C(X)$ such that $Z[\mathscr{A}]$ is a base for the closed subsets of $X$ and if $f \in \mathscr{A}$, then $|f| \in \mathscr{A}$. Then $\mathscr{A}$ is a Wallman ring on $X$.

Proof. It suffices to show that $F[\mathscr{A}]$ is Hausdorff (2.7). To show this we apply 2.4. Let $F, G \in F[\mathscr{A}], F \neq G$. Then $F \cap G$ is a $z$-filter ideal of $\mathscr{A}$ which is not prime. Using 4.1(3), we select $f, g \in \mathscr{A}$ such that $f g=0$, but $f \notin F \cap G$ and $g \notin F \cap G$. But $F$ and $G$ are prime ideals of $\mathscr{A}(1.7)$; hence, either $f \in F$ or $g \in F$. Suppose $f \in F$. Then $g \notin F$ and $f \notin G$. Also, if $g \in F$, then $f \notin F$ and $g \notin G$. By 2.4, then, $F[\mathscr{A}]$ is Hausdorff. Hence, $\mathscr{A}$ is a Wallman ring on $X$.

Corollary 4.3. Let $T \in c X$. Then $Z[E(X, T)]$ is a Wallman base for $X$. 


\section{REFERENCES}

1. C. M. Biles, Wallman-type compactifications, Ph. D. thesis, University of New Hampshire, November, 1968.

2. R. M. Brooks, On Wallman compactifications, Fund. Math. 40 (1967), 157-63.

3. O. Frink, Compactifications and semi-normal spaces, Amer. J. Math. 86 (1964), 602-607.

4. L. Gillman and M. Jerison, Rings of Continuous Functions, Van Nostrand, 1960.

5. A. Hager, On inverse-closed subalgebras of $C(X)$, Proc. London Math. Soc. (3) 19 (1969), 233-57.

6. E. Steiner, Wallman spaces and compactifications, Fund. Math. 61 (1968), 295-304.

7. E. Steiner and A. Steiner, Wallman and Z-compactifications, Duke Math. J. 35 (1968), 269-76.

Received September 11, 1969.

Humboldt State College 


\section{PACIFIC JOURNAL OF MATHEMATICS}

\section{EDITORS}

\author{
H. SAMELSON \\ Stanford University \\ Stanford, California 94305

\section{Richard Pierce} \\ University of Washington \\ Seattle, Washington 98105
}

J. DugundJI

Department of Mathematics

University of Southern California

Los Angeles, California 90007

RichaRd ARENS

University of California

Los Angeles, California 90024

\section{ASSOCIATE EDITORS}
E. F. BECKENBACH
B. H. NeUMANN
F. WOLE
K. YoshidA

\section{SUPPORTING INSTITUTIONS}

\author{
UNIVERSITY OF BRITISH COLUMBIA \\ CALIFORNIA INSTITUTE OF TECHNOLOGY \\ UNIVERSITY OF CALIFORNIA \\ MONTANA STATE UNIVERSITY \\ UNIVERSITY OF NEVADA \\ NEW MEXICO STATE UNIVERSITY \\ OREGON STATE UNIVERSITY \\ UNIVERSITY OF OREGON \\ OSAKA UNIVERSITY \\ UNIVERSITY OF SOUTHERN CALIFORNIA
}

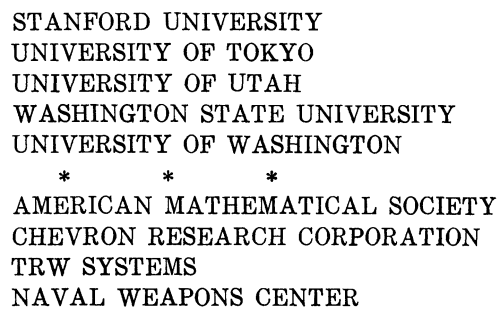

The Supporting Institutions listed above contribute to the cost of publication of this Journal, but they are not owners or publishers and have no responsibility for its content or policies.

Mathematical papers intended for publication in the Pacific Journal of Mathematics should be in typed form or offset-reproduced, (not dittoed), double spaced with large margins. Underline Greek letters in red, German in green, and script in blue. The first paragraph or two must be capable of being used separately as a synopsis of the entire paper. The editorial "we" must not be used in the synopsis, and items of the bibliography should not be cited there unless absolutely necessary, in which case they must be identified by author and Journal, rather than by item number. Manuscripts, in duplicate if possible, may be sent to any one of the four editors. Please classify according to the scheme of Math. Rev. Index to Vol. 39. All other communications to the editors should be addressed to the managing editor, Richard Arens, University of California, Los Angeles, California, 90024.

50 reprints are provided free for each article; additional copies may be obtained at cost in multiples of 50 .

The Pacific Journal of Mathematics is published monthly. Effective with Volume 16 the price per volume (3 numbers) is $\$ 8.00$; single issues, $\$ 3.00$. Special price for current issues to individual faculty members of supporting institutions and to individual members of the American Mathematical Society: $\$ 4.00$ per volume; single issues $\$ 1.50$. Back numbers are available.

Subscriptions, orders for back numbers, and changes of address should be sent to Pacific Journal of Mathematics, 103 Highland Boulevard, Berkeley, California, 94708.

PUBLISHED BY PACIFIC JOURNAL OF MATHEMATICS, A NON-PROFIT CORPORATION

Printed at Kokusai Bunken Insatsusha (International Academic Printing Co., Ltd.), 7-17, Fuj̣imi 2-chome, Chiyoda-ku, Tokyo, Japan. 


\section{Pacific Journal of Mathematics}

\section{Vol. 35, No. $2 \quad$ October, 1970}

Valentin Danilovich Belousov and Palaniappan L. Kannappan, Generalized Bol functional equation .................................... 259

Charles Morgan Biles, Gelfand and Wallman-type compactifications ........... 267

Louis Harvey Blake, A generalization of martingales and two consequent convergence theorems .................................... 279

Dennis K. Burke, On p-spaces and $w \Delta$-spaces..................... 285

John Ben Butler, Jr., Almost smooth perturbations of self-adjoint operators . . . . . . 297

Michael James Cambern, Isomorphisms of $C_{0}(Y)$ onto $C(X) \ldots \ldots \ldots \ldots \ldots . \ldots 307$

David Edwin Cook, A conditionally compact point set with noncompact closure ... 313

Timothy Edwin Cramer, Countable Boolean algebras as subalgebras and homomorphs .........................................

John R. Edwards and Stanley G. Wayment, A v-integral representation for linear operators on spaces of continuous functions with values in topological vector spaces.............................................

Mary Rodriguez Embry, Similarities involving normal operators on Hilbert

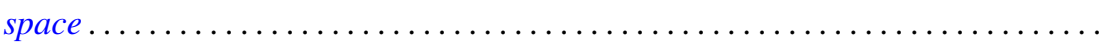

Lynn Harry Erbe, Oscillation theorems for second order linear differential

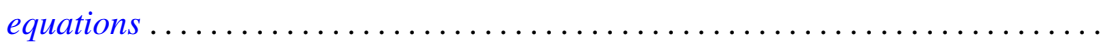

William James Firey, Local behaviour of area functions of convex bodies .......... Joe Wayne Fisher, The primary decomposition theory for modules ..............

Gerald Seymour Garfinkel, Generic splitting algebras for Pic ..................

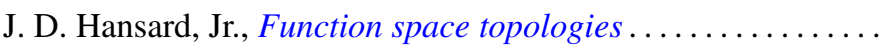

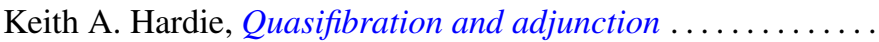

G. Hochschild, Coverings of pro-affine algebraic groups ...........

Gerald L. Itzkowitz, On nets of contractive maps in uniform spaces ..

381

389

399

417

Melven Robert Krom and Myren Laurance Krom, Groups with free nonabelian subgroups....................................

James Robert Kuttler, Upper and lower bounds for eigenvalues by finite differences ......................................

Dany Leviatan, A new approach to representation theory for convolution transforms . . .

Richard Beech Mansfield, Perfect subsets of definable sets of real numbers ...

Brenda MacGibbon, A necessary and sufficient condition for the embedding of a

Lindelof space in a Hausdorff $\mathscr{H} \sigma$ space ..................

David G. Mead and B. D. McLemore, Ritt's question on the Wronskian ....

Edward Yoshio Mikami, Focal points in a control problem .....

Paul G. Miller, Characterizing the distributions of three independent n-dimensional random variables, $X_{1}, X_{2}, X_{3}$, having analytic characteristic functions by the joint distribution of $\left(X_{1}+X_{3}, X_{2}+X_{3}\right)$. . .

P. Rosenthal, On the Bergman integral operator for an elliptic partial differential equation with a singular coefficient....

Douglas B. Smith, On the number of finitely generated $O$-group 